\title{
Denervación Coxofemoral con Abordaje Mínimamente Invasivo para el Control de Dolor en Perros con Enfermedad Degenerativa Articular
}

\author{
Coxofemoral Denervation with a Minimally Invasive Approach to Relieve Pain in \\ Dogs with Degenerative Joint Disease
}

Alvaro Luzio Q. ${ }^{1,2}$, Adrián Henríquez G. ${ }^{1}$, Christof Fischer W. ${ }^{1}$

\section{Resumen}

\begin{abstract}
La displasia de cadera corresponde a una incongruencia articular entre la cabeza femoral y el acetábulo, que usualmente evoluciona a enfermedad degenerativa articular (EDA), con signos de dolor crónico. Una opción quirúrgica para disminuir el dolor en pacientes con EDA es la denervación pericapsular de la articulación coxofemoral. En el presente estudio se realizó esta técnica a través de un abordaje mínimamente invasivo en 10 perros Pastor Alemán diagnosticados con displasia de cadera y EDA a través de un examen ortopédico e imagenología radiográfica. Para evaluar la eficacia de la técnica se aplicó una escala de dolor en el pre-quirúrgico y en los días 5,10 y 15 pos-quirúrgico. Los resultados demostraron una disminución efectiva del dolor entre los días 0 y 15 y entre los días 5 y 15 . Así también, se evidenció una percepción favorable por parte de los propietarios, donde seis de ellos indicaron que la cirugía resultó ser satisfactoria y los otros cuatro indicaron que la cirugía fue buena, en tanto que todos ellos dijeron que volverían a realizar la cirugía a sus mascotas. Se concluye que es posible disminuir el dolor con una denervación coxofemoral, mediante un abordaje mínimamente invasivo en pacientes con EDA.
\end{abstract}

Palabras clave: denervación coxofemoral; enfermedad degenerativa articular; perros

\section{Abstract}

Hip dysplasia can be defined as a joint incongruity between the femoral head and the acetabulum, which usually develops to degenerative joint disease (DJD), with signs of chronic pain. A surgical option to reduce pain in patients with EDA is the pericapsular

\footnotetext{
${ }^{1}$ Escuela de Medicina Veterinaria, Facultad de Recursos Naturales y Medicina Veterinaria, Universidad Santo Tomás, Concepción, Chile

${ }^{2}$ E-mail: aluzio@santotomas.cl
}

Recibido: 18 de abril de 2017

Aceptado para publicación: 29 de julio de 2017 
denervation of the hip joint. In the present study, this technique was performed through a minimally invasive approach in 10 German Shepherd dogs diagnosed with hip dysplasia and EDA through an orthopedic examination and radiographic imaging. To assess the efficacy of the technique a pain scale was applied in the pre-surgical on days 5,10 and 15 post-surgery. The results showed an effective decrease of pain between days 0 and 15 and between days 5 and 15 . Also, a favorable perception was evidenced by the owners, where six of them indicated that the surgery proved to be satisfactory and the others four indicated that the surgery was good, while all of them said they would request again the surgery to their pets. It is concluded that it is possible to reduce pain with a coxofemoral denervation through a minimally invasive approach in patients with EDA.

Key words: coxofemoral denervation; degenerative joint disease; dogs

\section{INTRODUCCIÓN}

La displasia de cadera es una enfermedad del desarrollo, de observación frecuente, que produce dolor articular en perros (Johnson et al., 1994). Las causas son multifactoriales, incluidos factores hereditarios, ambientales, nutricionales y hormonales (Selmi et al., 2009). Además, existe una clara predisposición racial, ya que la enfermedad es típica de razas grandes, donde se observa un crecimiento acelerado durante los primeros meses de vida, produciendo una excesiva laxitud entre el fémur y la cadera (Johnson et al., 1994).

En edades avanzadas, en la misma articulación y producto de la displasia, se genera una Enfermedad Degenerativa Articular (EDA) progresiva (Dassler, 2003). La historia de los signos clínicos incluye anormalidades al andar, tales como claudicación, intolerancia al ejercicio, dificultad al levantarse y subir escaleras (Dassler, 200). Se han descrito dos modalidades de tratamiento para esta patología: el conservador y el quirúrgico (Tomlinson y Mclaughlin, 1996; Hulse y Johnson, 2002).

Dentro de los tratamientos quirúrgicos se encuentra la denervación pericapsular coxofemoral percutánea, un procedimiento para etapas avanzadas de la displasia y su consecuente EDA. Esta última técnica se basa en la denervación de la cápsula periarticular coxofemoral obtenida por la destrucción de ramas nerviosas articulares, cuyo objetivo es reducir el dolor y devolver el movimiento a la articulación (Kinzel et al., 2002; Schmaedecke et al., 2008). El presente estudio tuvo como objetivo describir una técnica quirúrgica mínimamente invasiva de denervación pericapsular coxofemoral en perros con EDA coxofemoral y determinar si dicho procedimiento es eficaz en disminuir el dolor en caninos afectados con esta patología.

\section{Materiales y Métodos}

\section{Estandarización de la Técnica Quirúrgica}

En una primera etapa del estudio, se estandarizó la técnica con abordaje mínimamente invasivo en seis articulaciones coxofemorales de cadáveres caninos, de tamaño grande, similares a los utilizados en el estudio.

- Se depiló la zona topográfica coxofemoral, comprendida entre el proceso isquiático, la cresta iliaca y el trocánter mayor del fémur, de tal manera de generar un triángulo imaginario entre los tres puntos, siendo el más ventral la región anatómica del trocánter mayor del fémur. 
- Desde el centro de este último punto, se trazó una línea en dirección cráneo dorsal hasta llegar a la cresta iliaca.

- Desde el trocánter mayor a una distancia de $1 / 3$ de la línea antes trazada en dirección cráneo dorsal, se realizó una punción con una aguja hipodérmica de $18 \mathrm{G}$ x 1", hasta llegar a la porción cráneo dorsal de la ceja del acetábulo e inyectar tinta de color negro para marcar la zona exacta sobre el hueso (Figura 1).

- Luego se procedió a efectuar una incisión sobre la piel en la zona donde se insertó la aguja, llegando a los $10 \mathrm{~cm}$ de extensión y en profundidad atravesando subcutáneo, músculo tensor de la fascia lata, el músculo glúteo medio, inserción del músculo basto lateral y, por último, el músculo glúteo profundo, que dejó despejado el músculo gemelo craneal (Sisson y Grossman, 1998).

- De esta manera quedó expuesta la porción ósea de la cadera para evidenciar y verificar si la punción con la aguja ocurrió exactamente en la región cráneo dorsal de la ceja acetabular, que se condice con la zona anatómica donde se inserta la inervación sensitiva correspondiente a las ramas de los nervios glúteos craneales e isquiático (Schmaedecke et al., 2008).

- Luego se realizó el mismo procedimiento, pero esta vez efectuando una incisión de $1 \mathrm{~cm}$ de extensión en la otra hemicadera del cadáver, sobre el mismo punto topográfico antes señalado, pero sin inyectar el tinte negro, e ingresando directamente con un clavo de Steinmann de $8 \mathrm{~mm}$ a la zona cráneo dorsal.

- Una vez sobre el periostio, se realizaron 15 movimientos de raspado en orientación caudo craneal y luego dorso ventral, cubriendo el tercio cráneo dorsal completo y sin separar la punta del clavo de Steinmann de la cadera en ningún momento para evitar un daño sobre el nervio isquiático.

- Se repitió el procedimiento anterior que comprende la extensión de la incisión primaria hasta los $10 \mathrm{~cm}$, llegando a despejar completamente la región ósea en cuestión para evidenciar y verificar el lugar exacto donde se realizó la desperiostización.

- Este último fue el lugar exacto donde se efectuó la denervación en pacientes diagnosticados con EDA.

\section{Determinación de la Artralgia de la Arti- culación Coxofemoral}

En la segunda etapa del estudio se incluyeron 10 caninos Pastor Alemán. Todos los pacientes evaluados tenían más de un año de edad y mostraban un proceso doloroso, ya sea poco apetito, disminución en su actividad física o restricción de ciertos movimientos (Firth y Haldane, 1999).

Se realizó la anamnesis de los pacientes con EDA y una evaluación ortopédica de cada individuo para evidenciar alteraciones en la marcha, dolor en la flexión, extensión, abducción del miembro y en la palpación de la articulación coxofemoral. A los pacientes que mostraron evidencias clínicas de alteraciones en la articulación coxofemoral se les realizó un examen radiográfico de cadera para confirmar alteraciones degenerativas coxofemorales.

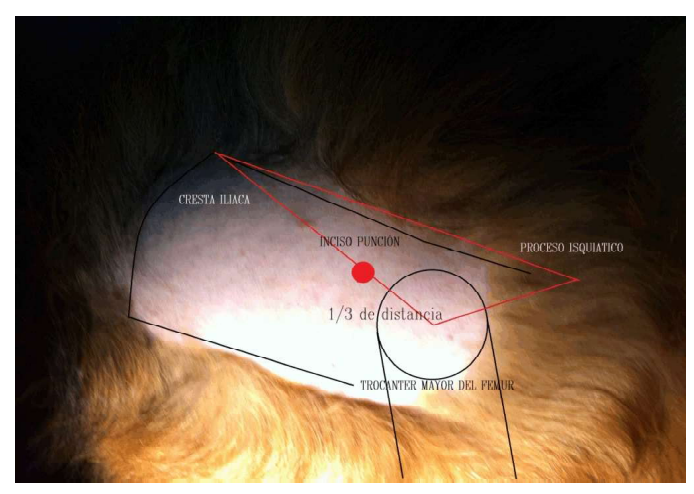

Figura 1. Relaciones topográficas descritas sobre una hemicadera izquierda en un cadáver canino. El punto rojo indica el lugar de la inciso-punción. 
Cuadro 1. Escala de medición del dolor, modificada de Autefage et al. (2011)

\begin{tabular}{llc}
\hline Signos clínicos Descripción & Puntaje \\
\hline Claudicación & Ausente & 0 \\
& Leve & 1 \\
& Moderada & 2 \\
& Severa & 3 \\
\hline Reacción a & Sin reacción & 0 \\
palpación & Leve & 1 \\
& Moderada & 2 \\
& Severa & 3 \\
\hline Rango de & Normal & 0 \\
movimiento & Levemente & 1 \\
& reducida & \\
& Moderadamente & 2 \\
& reducida & \\
& Severamente & 3 \\
& reducida & \\
\hline
\end{tabular}

Los 10 pacientes fueron sometidos a la primera medición de grados de dolor, evaluado mediante una escala modificada de dolor crónico (Cuadro 1) (Autefage et al., 2011). Los valores obtenidos en cada una de las tres categorías se sumaron y se obtuvo el valor total de la escala para cada paciente. El rango de valores puede ser entre 0 y 9 , donde 0 es ausencia de dolor, entre 1 y 3 es dolor leve, entre 4 y 6 es dolor moderado y entre 7 y 9 es dolor severo (Autefage et al., 2011). Estas evaluaciones fueron hechas por un mismo médico veterinario.

La intervención quirúrgica de los pacientes fue de la manera descrita para la estandarización de la técnica quirúrgica. Todos los pacientes fueron operados de manera bilateral. Las evaluaciones del dolor se valoraron en tres tiempos posquirúrgicos (días $5,10$ y 15$)$.

\section{Percepción de los Propietarios}

Luego de las mediciones posquirúrgicas se realizó una encuesta a los propietarios para conocer sus percepciones respecto del resultado clínico de la técnica quirúrgica, mediante un cuestionario (modificado de Mölsä et al., 2013) (Cuadro 2).

\section{Análisis Estadístico}

Se utilizó el test de Friedman para la comparación entre los tiempos de evaluación, considerando una diferencia estadística significativa con valores de $\mathrm{p}<0.05$, y se utilizó el test de comparaciones múltiples de Dunns para identificar los tiempos que fueron estadísticamente diferentes.

\section{Resultados}

En la inspección de las dos primeras caderas intervenidas se pudo evidenciar la precisión de los parámetros topográficos predispuestos, ya que en ambas ocasiones se logró desperiostizar la zona exacta. Los parámetros topográficos externos siguieron siendo muy precisos en las siguientes cuatro hemicaderas, donde se mejoró la extensión y la profundidad del curetaje, procurando evitar separar el periostio del clavo de Steinmann.

No se observaron variaciones en cuando a la zona tratada al despejar la articulación, pero se observaron mejoras respecto de la calidad del curetaje. De igual manera, no se observaron daños en estructuras importantes como el nervio isquiático. Al obtener exactamente el mismo resultado en la última hemicadera se dio por estandarizada la técnica quirúrgica.

En la evaluación del dolor pre-quirúrgico de los 10 perros con EDA, ocho presentaron puntuaciones entre 4 a 6 (dolor moderado) y dos presentaron puntuaciones de 7 y 8 (dolor severo), los cuales, a su vez, resultaron ser los pacientes con mayor edad (8 y 9 años, respectivamente) (Cuadro 3). 
Cuadro 2. Percepción de los propietarios respecto al resultado de la cirugía de denervación pericapsular coxofemoral en perros con EDA coxofemoral. Cuestionario modificado de Mölsä et al. (2013)

¿Como diría usted que fue el resultado de la cirugía de cadera en su mascota?
a) Bueno:
Sin cojera, apoyo del peso completo sobre miembros al caminar, trotar, correr y saltar. Buen apetito.
b) Satisfactorio:
Leve cojera y rigidez ocasional de vez en cuando. Realiza actividad física moderada. Apetito moderado.
c) Insatisfactorio:
Cojera permanente y acentuada en frío y pos-ejercicio físico.
Cojera al caminar, rotar, correr y saltar. Mal apetito.

Si se diera la posibilidad, ¿Volvería a realizar esta cirugía a su mascota u otra mascota que lo necesitara?

- Si, de todas maneras

- Tal vez, lo pensaría

- No volvería a realizarla en alguna mascota

Cuadro 3. Resultados de las evaluaciones del dolor en la fase pre (día 0 ) y pos-quirúrgica en 10 perros con enfermedad degenerativa articular

\begin{tabular}{ccccc}
\hline Paciente & Día 0 & Día 5 & Día 10 & Día 15 \\
\hline 1 & 5 & 4 & 3 & 2 \\
2 & 6 & 7 & 3 & 2 \\
3 & 5 & 7 & 2 & 2 \\
4 & 8 & 7 & 5 & 4 \\
5 & 6 & 5 & 5 & 4 \\
6 & 6 & 6 & 4 & 3 \\
7 & 5 & 6 & 5 & 4 \\
8 & 6 & 6 & 5 & 4 \\
9 & 7 & 6 & 4 & 3 \\
10 & 4 & 4 & 3 & 3 \\
\hline Promedio & $5.8^{\mathrm{a}}$ & $5.8^{\mathrm{a}}$ & $3.9^{\mathrm{a}}$ & $3.1^{\mathrm{b}}$ \\
\hline
\end{tabular}

a,b Superíndices diferentes indican diferencia estadística entre periodos de evaluación ( $p=0.0001)$

Los ocho pacientes que presentaban dolor moderado presentaron baja reacción dolorosa a la palpación de la zona coxofemoral, no así en la manipulación del miembro, buscando su máxima amplitud articular en donde mostraban claras evidencia de dolor; ya sea vocalizando, mirando la zona afectada o manifestándose mediante mordidas. Los perros con dolor severo se mostraron particularmente sensibles a la palpación de la articulación coxofemoral, lo que elevó el puntaje final de la evaluación incidiendo en su categorización final. 
Las evaluaciones pre-quirúrgicas fueron la base para la comparación con las evaluaciones pos- quirúrgicas (Cuadro 3). En el día 5 pos-quirúrgico, tres pacientes aumentaron 1 punto, cuatro bajaron 1 punto y tres perros mantuvieron el puntaje pre-quirúrgico. Todos los pacientes manifestaron disminución del dolor en el día 10 pos-quirúrgico, mientras que los cambios fueron evidentes en la evaluación del día 15 pos-quirúrgico, donde seis pacientes bajaron a la categoría dolor leve y los otros cuatro se mantuvieron en la categoría dolor moderado, pero bajando 1 punto dentro de la misma categoría. Se observaron diferencias significativas entre los días 0 con el día 15 , no encontrándose diferencias estadísticas al comparar el día 0 con los días 5 y 10.

La mayoría de los propietarios $(6 / 10)$ describió el procedimiento como satisfactorio y los demás $(4 / 10)$ como bueno. Por otro lado, todos los propietarios afirmaron que volverían a solictar la cirugía frente a la presencia de una situación similar.

\section{Discusión}

La técnica quirúrgica descrita en el presente trabajo presenta todas las características de una intervención mínimamente invasiva, ya que se disminuyó la incisión cutánea, se modificó el acceso quirúrgico buscando disminuir las lesiones tisulares, se disminuyeron las disecciones profundas, se conservó la mayor cantidad de tejido muscular y se utilizó material que respetó los tejidos blandos asociados a la zona en cuestión (Varela et al., 2010). Por otro lado, los tiempos anestésicos y quirúrgicos fueron reducidos, siendo el tiempo máximo de duración de cada cirugía de 10 minutos desde la inducción del animal, lo que permite utilizar anestésicos de rápida acción y corta duración, reduciendo los tiempos anestésicos y, por ende, los riesgos asociados a alteraciones neurológicas, cardiovasculares y respiratorias en el paciente (Collard et al., 2010).
En la técnica tradicional se emplea un abordaje abierto realizando una incisión de 7 $\mathrm{cm}$ de extensión despejando la musculatura involucrada, se apoya con separadores para obtener un campo visual amplio y realizar la desperiostización con curetas a lo largo de todo el borde acetabular, para finalmente suturar la musculatura, el subcutáneo y la piel (Ferrigno et al., 2007). Este procedimiento implica un tiempo quirúrgico y anestésico más prolongado, además de aumentar los estímulos dolorosos para el paciente y un tiempo de recuperación más largo.

A diferencia de la técnica clásica, la técnica empleada en este estudio reduce la incisión de $7 \mathrm{~cm}$ a una inciso-punción de $1 \mathrm{~cm} \mathrm{y}$ sin intervenir mayormente la integridad de grupos musculares, lo que reduce los tiempos quirúrgicos, anestésicos y el tiempo de recuperación pos-quirúrgico. Probablemente, la mayor dificultad de la técnica quirúrgica no invasiva sea dar con la zona exacta para realizar la denervación, de ahí la importancia de las referencias anatómicas establecidas en la estandarización e identificación de estas al momento de realizar la cirugía. Estas mismas referencias anatómicas se repiten en los estudios donde se ha realizado la denervación coxofemoral (Minto et al., 2012), pero sin realizar un abordaje mínimamente invasivo, por lo que el abordaje se hace más fácil al visualizar las estructuras anatómicas con mayor claridad.

Los resultados obtenidos en este estudio son similares a los expuestos en el estudio de Ferrigno et al. (2007) utilizando una técnica quirúrgica más invasiva, aun cuando en dicho estudio cerca del $95 \%$ de los pacientes presentaron una disminución significativa del dolor en los días 7 y 14 pos-quirúrgicos respecto al pre-quirúrgico, y los cambios observados hasta el año de la cirugía no fueron mayormente diferentes con respecto al día 14. De igual forma, Collard et al. (2010) trabajando con nueve perros con EDA obtuvieron resultados similares al estudio anterior, reduciendo eficazmente el dolor en el día 
15 pos-quirúrgico, y sin observar diferencias entre las evaluaciones de los días 15 y 180.

Considerando los resultados de los trabajos de Collard et al. (2010) y Ferrigno et al. (2007), donde se evidencian cambios significativos en el día 15 pos-quirúrgico con relación al tiempo cero, permite deducir que el día 15 es el momento adecuado para la evaluación del dolor pos-quirúrgico en este tipo de intervención quirúrgica. Es probable que la punción en el tejido muscular generada en la cirugía haya provocado la presencia de dolor, que no alcanzó a ser controlado antes de los 5 días posteriores a la cirugía.

Con respecto a la percepción de los propietarios, resultados similares se obtuvieron en otros estudios. Ferrigno et al. (2007) indican que el $72.2 \%$ de los propietarios a los 14 días de la cirugía calificaron como satisfactorios los resultados en sus mascotas e indicaron una evidente mejoría en su calidad de vida. De la misma forma, Minto et al. (2012) realizaron la denervación acetabular en 15 perros cuya evaluación se basó únicamente en una escala de percepción del propietario a los días 7, 30 y 60 pos-quirúrgico, indicando que al día 7 todos los propietarios consideraban los resultados de la cirugía como buenos, y que al día 30 solo uno (3\%) de los pacientes presentaba un grado leve de claudicación y los propietarios categorizaban el resultado de la cirugía como óptima. En este trabajo, al igual que en los mencionados anteriormente, todos los propietarios indicaron una mejor calidad de vida en sus mascotas al día 15 de la cirugía. Las principales observaciones de los propietarios se relacionan con recuperación del apetito, mayor actividad en general y menor grado subjetivo de claudicación.

\section{Conclusiones}

La denervación pericapsular con un abordaje mínimamente invasivo es efectiva para reducir el dolor en perros con degeneración articular coxofemoral.
Literatura Citada

1. Autefage A, Palissier FM, Asimus E, Pepin-Richard C. 2011. Long-term efficacy and safety of firocoxib in the treatment of dogs with osteoarthritis. Vet Rec 168: 617-617. doi: 10.1136/vr.d1456

2. Collard F, Maitre P, Le Quang T, Fau $D$, Carozzo C, Genevoist J-P, et al. 2010. Canine hip denervation: comparison between clinical outcome and gait analysis. Revue Med Vet 161: 277-282.

3. Dassler CL. 2003. Canine hipdysplasia: diagnosis and nonsurgical treatment. In: Slatter D (ed). Textbook of small animal surgery. Philadelphia, USA: Saunders. $p$ 2019-2020.

4. Ferrigno CR, Schmaedecke A, Oliveira LM, D'Ávila RS, Yamamoto EY, et al. 2007. Denervação acetabular cranial e dorsal no tratamento da displasia coxofemoral em cães: 360 dias de evolução de 97 casos. Pesq Vet Bras 27: 333-340. doi: 10.1590/S0100-736X2007000800003

5. Firth A, Haldane S. 1999. Development of a scale to evaluate postoperative pain in dogs. J Am Vet Med Assoc 214: 651-659.

6. Hulse D, Johnson A. 2002. Tratamiento de la enfermedad articular. En: Fossum TW (ed). Cirugía de pequeños animales. Buenos Aires, Argentina: Meìdica Panamericana. p 1042-1050.

7. Johnson JA, Austin C, Breur GJ. 1994. Incidence of canine appendicular musculoskeletal disorders in 16 veterinary teaching hospitals from 1980 through 1989. Vet Comp Orthop Traumatol 7: 56-69.

8. Kinzel S, Hein S, von Scheven C, Küpper $\boldsymbol{W}$. 2002. 10 years experience with denervation of the hip joint capsule in the treatment of canine hip joint dysplasia and arthrosis. Berl Münch Tierärztl Wochenschr 115: 53-56. [En alemán]. 
9. Minto BW, Souza VLD, Brandão CVS, Mori ES, Morishin Filho M, Ranzani JJT. 2012. Avaliação clínica da denervação acetabular em cães com displasia coxofemoral atendidos no hospital veterinário da FMVZ - Botucatu SP. Vet Zootec 19: 91-98.

10. Mölsä SH, Hielm-Björkman AK, Laitinen-Vapaavuori OM. 2013. Use of an owner questionnaire to evaluate long-term surgical outcome and chronic pain after cranial cruciate ligament repair in dogs: 253 cases (2004-2006). J Am Vet Med Assoc 243: 689-695. doi: 10.2460/javma.243.5.689

11. Schmaedecke A, Saut J, Ferrigno C. 2008. A quantitative analysis of the nerve fibres of the acetabular periosteum of dogs. Vet Comp Orthop Traumatol 5: 413-417.

12. Selmi A, Penteado B, Lins B. 2009. Denervação capsular percutânea no tratamento da displasia coxofemoral canina. Ciência Rural 39: 460-466.

13. Sisson S, Grossman JM. 1998. Anatomía de los animales domésticos. Barcelona: Mediterraneo. $1414 \mathrm{p}$.

14. Tomlinson J, Mclaughlin R. 1996. Medically managing canine hip dysplasia. Vet Med 91: 48-53.

15. Varela EJR, Suárez MÁ, Fernández M, González, V, Varela JR, Murcia A. 2010. Abordaje posterior mínimamente invasivo en artroplastia total de cadera. Estudio prospectivo y aleatorizado. Un año de seguimiento. Anales Sin Sas Navarra 33: 133-143. 